Pacific

Journal of

Mathematics

\title{
CERTIFYING INCOMPRESSIBILITY \\ OF NONINJECTIVE SURFACES WITH SCL
}

DANNY CALEGARI

Volume 262

No. 2

April 2013 


\title{
CERTIFYING INCOMPRESSIBILITY OF NONINJECTIVE SURFACES WITH SCL
}

\author{
DANNY CALEGARI
}

\begin{abstract}
Cooper and Manning (2011) and Louder (2011) gave examples of maps of surface groups to $\operatorname{PSL}(2, \mathbb{C})$ which are not injective, but are incompressible (i.e., no simple loop is in the kernel). We construct more examples with very simple certificates for their incompressibility arising from the theory of stable commutator length.
\end{abstract}

The purpose of this note is to give examples of maps of closed surface groups to $\operatorname{PSL}(2, \mathbb{C})$ which are not $\pi_{1}$-injective, but are geometrically incompressible, in the sense that no simple loop in the surface is in the kernel (in the sequel we use the word "incompressible" as shorthand for "geometrically incompressible"). The examples are very explicit, and the images can be taken to be all loxodromic. The significance of such examples is that they shed light on the simple loop conjecture, which says that any noninjective map from a closed oriented surface to a 3-manifold should be compressible.

Examples of such maps were first shown to exist in [Cooper and Manning 2011], by a representation variety argument, thereby answering a question of Minsky [2000] (also see [Bowditch 1998]). More sophisticated examples were then found by Louder [2011]; he even found examples with the property that the minimal self-crossing number of a loop in the kernel can be taken to be arbitrarily large. Louder's strategy is to exhibit an explicit finitely presented group (a limit group) which admits noninjective incompressible surface maps, and then to observe that such a group can be embedded as an all-loxodromic subgroup of $\operatorname{PSL}(2, \mathbb{C})$.

It is easy to produce examples of noninjective surface groups. What is hard is to certify that they are incompressible. The main point of our construction, and the main novelty and interest of this paper, is to show that stable commutator length (and its cousin Gromov-Thurston norm) can be used to certify incompressibility.

Our examples are closely related to Louder's examples, although our certificates are quite different. So another purpose of this note is to advertise the use of stable

Danny Calegari was partly supported by NSF grant DMS 1005246.

MSC2010: 20F12, 57M60.

Keywords: simple loop conjecture, stable commutator length, compressible, PSL(2,C). 
commutator length as a tool to get at the kind of information that is relevant in certain contexts in the theory of limit groups.

We move back and forward between (fundamental) groups and spaces in the usual way. We assume the reader is familiar with stable commutator length, and Gromov-Thurston norms in dimension 2. Standard references are [Calegari 2009; Gromov 1982; Thurston 1986]. Computations are done with the program scallop, available from [Calegari and Walker 2011].

Recall that if $X$ is a $K(\pi, 1)$, the Gromov-Thurston norm of a class $\alpha \in H_{2}(X ; \mathbb{Z})$ (denoted $\|\alpha\|$ ) is the infimum of $-\chi(T) / n$ over all closed, oriented surfaces $T$ without spherical components mapping to $X$ and representing $n \alpha$. Our certificates for incompressibility are guaranteed by the following proposition.

Proposition 1 (certificate). Let $X$ be a $K(\pi, 1)$, and let $\alpha \in H_{2}(X ; \mathbb{Z})$ be represented by a closed oriented surface $S$ with no torus or spherical components. If there is a strict inequality $\|\alpha\|>-\chi(S)-2$ (where $\|\cdot\|$ denotes Gromov-Thurston norm) then $S$ is (geometrically) incompressible.

Proof. If $S$ is compressible, then $\alpha$ is represented by the result of compressing $S$, which is a surface $S^{\prime}$ with no spherical components, and $-\chi\left(S^{\prime}\right)<\|\alpha\|$. But this contradicts the definition of $\|\alpha\|$.

On the other hand, a closed surface $S$ without torus or spherical components representing $\alpha$ and with $-\chi(S)=\|\alpha\| i$ is $\pi_{1}$-injective, so to apply our proposition to obtain examples, we must find examples of spaces $X$ and integral homology classes $\alpha$ for which $\|\alpha\|$ is not equal to $-\chi(S)$ for any closed orientable surface $S$; i.e., for which $\|\alpha\|$ is not in $2 \mathbb{Z}$. Such spaces can never be 3-manifolds, by combined results of [Gabai 1983; Thurston 1986], so our methods will never directly find a counterexample to the simple loop conjecture.

The groups we consider are all obtained by amalgamating two simpler groups over a cyclic subgroup. The generator of the cyclic group is homologically trivial in either factor, giving rise to a class in $H_{2}$ in the big group. The Gromov-Thurston norm of this class is related to the stable commutator length of the loop in the two factors as follows:

Proposition 2 (amalgamation). Let $G$ be an amalgamated product $G=J *_{\langle w\rangle} K$ along a cyclic group $\langle w\rangle$ which is generated by a loop $w$ which is homologically trivial on either side. Let $\phi: H_{2}(G ; \mathbb{Z}) \rightarrow H_{1}(\langle w\rangle ; \mathbb{Z})$ be the connecting map in the Mayer-Vietoris sequence, and let $H_{w} \subset H_{2}(G ; \mathbb{Z})$ be the affine subspace mapping to the generator. If $w$ has infinite order in $J$ and $K$, then

$$
\inf _{\alpha \in H_{w}}\|\alpha\|=2\left(\operatorname{scl}_{J}(w)+\operatorname{scl}_{K}(w)\right) .
$$

Proof. This is not difficult to see directly from the definition, and it is very similar to the proof of Theorem 3.4 in [Calegari 2008]. However, for the sake of clarity 
we give an argument. Note by the way that the hypothesis that $w$ is homologically trivial on either side is equivalent to the statement that the inclusion map $H_{1}(\langle w\rangle ; \mathbb{Z}) \rightarrow H_{1}(J ; \mathbb{Z}) \oplus H_{1}(K ; \mathbb{Z})$ is the zero map, so $\phi$ as above is certainly surjective. Moreover, if $H_{2}(J ; \mathbb{Z})$ and $H_{2}(K ; \mathbb{Z})$ are trivial (as will often be the case below), then $\phi$ is an isomorphism, and $H_{w}$ consists of a single class $\alpha$.

It is convenient to geometrize this algebraic picture, so let $X_{J}$ and $X_{K}$ be Eilenberg-MacLane spaces for $J$ and $K$, and let $X_{G}$ be obtained from $X_{J}$ and $X_{K}$ by attaching the two ends of a cylinder $C$ to loops representing the conjugacy classes corresponding to the images of $w$ in either side. Let $\gamma$ be the core of $C$. If $S$ is a closed, oriented surface with no sphere components, and $f: S \rightarrow X_{G}$ represents some $n \alpha$ with $\alpha \in H_{w}$, then we can homotope $f$ so that it meets $\gamma$ transversely and efficiently - i.e., so that $f^{-1}(\gamma)$ consists of pairwise disjoint essential simple curves in $S$. If one of these curves maps to $\gamma$ with degree zero we can compress $S$ and reduce its complexity, so without loss of generality every component maps with nonzero degree. Hence we can cut $S$ into $S_{J}$ and $S_{K}$ each mapping to $X_{J}$ and $X_{K}$ respectively and with boundary representing some finite cover of $w$. By definition this shows $\inf _{\alpha \in H_{w}}\|\alpha\| \geq 2\left(\operatorname{scl}_{J}(w)+\operatorname{scl}_{K}(w)\right)$.

Conversely, given surfaces $S_{J}$ and $S_{K}$ mapping to $X_{J}$ and $X_{K}$ with boundary representing finite covers of $w$ (or rather its image in each side), we need to construct a suitable $S$ as above. First, we can pass to a cover of each $S_{J}$ and $S_{K}$ in such a way that the boundary of each maps to $w$ with positive degree; see, for example, Proposition 2.13 of [Calegari 2009]. Then we can pass to a further finite cover of each so that the set of degrees with which components of $\partial S_{J}$ and of $\partial S_{K}$ map over $w$ are the same (with multiplicity); again, see the argument of the proposition just cited. Once this is done we can glue up $S_{J}$ to $S_{K}$ with the opposite orientation to build a surface $S$ mapping to $X_{G}$ which, by construction, represents a multiple of some $\alpha$ in $H_{w}$. We therefore obtain $\inf _{\alpha \in H_{w}}\|\alpha\| \leq 2\left(\operatorname{scl}_{J}(w)+\operatorname{scl}_{K}(w)\right)$ and we are done.

We now show how to use these propositions to produce examples.

Example 1. Start with a free group; for concreteness, let $F=\langle a, b, c\rangle$. Consider a word $w \in F$ of the form $w=[a, b][c, v]$ for some $v \in F$. Associated to this expression of $w$ as a product of two commutators is a genus 2 surface $S$ with one boundary component mapping to a $K(F, 1)$ in such a way that its boundary represents $w$. This surface is not injective, since the image of its fundamental group is $F$ which has rank 3. Let $G=\langle a, b, c, x, y \mid w=[x, y]\rangle$; i.e., geometrically a $K(G, 1)$ is obtained from a $K(F, 1)$ by attaching the boundary of a once-punctured torus $T$ to $w$. The surface $R:=S \cup T$ has genus 3 , and represents the generator of $H_{2}(G ; \mathbb{Z})$. On the other hand, by the Amalgamation Proposition, the Gromov-Thurston norm of this homology class is equal to $2 \cdot \operatorname{scl}_{\langle x, y\rangle}([x, y])+2 \cdot \operatorname{scl}_{F}(w)$. Since $\operatorname{scl}_{\langle x, y\rangle}([x, y])=\frac{1}{2}$ 
(see [Calegari 2009, Example 2.100], for instance), providing $\frac{1}{2}<\operatorname{scl}(w)$ the result is noninjective but incompressible.

The group $G$ can be embedded in $\operatorname{PSL}(2, \mathbb{C})$ by first embedding $F$ as a discrete subgroup, then embedding $\langle x, y\rangle$ in such a way that $[x, y]=w$. By conjugating $\langle x, y\rangle$ by a generic loxodromic element with the same axis as $w$, we can ensure this example is injective, and it can even be taken to be all loxodromic. This follows in the usual way by a Bass-Serre type argument; a similar argument appears in [Calegari and Dunfield 2006, Lemma 1.5].

Almost any word $v$ will give rise to $w$ with $\operatorname{scl}(w)>\frac{1}{2}$; for example,

$$
\operatorname{scl}([a, b][c, a a])=1,
$$

as can be computed using scallop. Experimentally, it appears that if $v$ is chosen to be random of length $n$, then $\operatorname{scl}(w) \rightarrow \frac{3}{2}$ as $n \rightarrow \infty$. For example,

$$
\operatorname{scl}([a, b][c, b c A B B c A B C b b c A C b c B c b b])=\frac{7}{5} \text {. }
$$

The $\operatorname{closer} \operatorname{scl}(w)$ is to $\frac{3}{2}$, the bigger the index of a cover in which some simple loop compresses. This gives a practical method to produce examples for any given $k$ in which no loop with fewer than $k$ self-crossings is in the kernel.

Example 2. Note that the groups $G$ produced in Example 1 are 1-relator groups, which are very similar to 3-manifold groups in some important ways. A modified construction shows they can in fact be taken to be 1-relator fundamental groups of hyperbolic 4-manifolds. To see this, we consider examples of the form $G=$ $\left\langle a, b, c, x_{1}, y_{1}, \ldots, x_{g}, y_{g} \mid w=\prod_{i=1}^{g}\left[x_{i}, y_{i}\right]\right\rangle$ i.e., we attach a once-punctured surface $T_{g}$ of genus $g$, giving rise to a noninjective incompressible surface $R=S \cup T_{g}$ of genus $g+2$.

Let $\langle a, b, c\rangle$ act discretely and faithfully, stabilizing a totally geodesic $\mathbb{M}^{3}$ in $\mathbb{U}^{4}$. We can arrange for the axis $\ell$ of $w$ to be disjoint from its translates. Thinking of $\left\langle x_{1}, y_{1}, \ldots, x_{g}, y_{g}\right\rangle$ as the fundamental group of a once-punctured surface $T_{g}$, we choose a hyperbolic structure on this surface for which $\partial T_{g}$ is isometric to $\ell /\langle w\rangle$, and make this group act by stabilizing a totally geodesic $\mathbb{M}^{2}$ in $\mathbb{H}^{4}$ in such a way that the axis of $\partial T_{g}$ intersects the $\mathbb{H}^{3}$ perpendicularly along $\ell$. Providing the shortest essential arc in $T_{g}$ from $\partial T_{g}$ to itself is sufficiently long (depending on the minimal distance from $\ell$ to its translates by $\langle a, b, c\rangle$ ) the resulting group is discrete and faithful. This follows by applying the Klein-Maskit combination theorem, once we ensure that the limit sets of the conjugates of $\langle a, b, c\rangle$ are contained in regions satisfying the ping-pong hypothesis for the action of $\pi_{1}\left(T_{g}\right)$. This condition can be ensured by taking $g$ big enough and choosing the hyperbolic structure on $T_{g}$ accordingly; the details are entirely straightforward. 
Example 3. Let $H$ be any nonelementary hyperbolic 2-generator group which is torsion free but not free. Let $a, b$ be the generators. Then the once-punctured torus with boundary $[a, b]$ is not injective. As before, let $G=\langle H, x, y \mid[a, b]=[x, y]\rangle$. Then $G$ contains a genus 2 surface representing the amalgamated class in $H_{2}(G ; \mathbb{Z})$, and the norm of this class is $1+2 \cdot \operatorname{scl}_{H}([a, b])>0$, so this example is noninjective but incompressible.

As an example, we could take $H$ to be the fundamental group of a closed hyperbolic 3-manifold of Heegaard genus 2, or a 2-bridge knot complement. Such examples have discrete faithful representations into $\operatorname{PSL}(2, \mathbb{C})$.

Example 4. It is easy to produce examples of 2-generator 1-relator groups $H=$ $\langle a, b \mid v\rangle$ in which $\frac{1}{2}-\epsilon<\operatorname{scl}([a, b])<\frac{1}{2}$ for any $\epsilon$. Such groups are torsion-free if $v$ is not a proper power. Just fix some big integer $N$ and take

$$
v=\left([a, b]^{ \pm N}\right)^{g_{1}}\left([a, b]^{ \pm N}\right)^{g_{2}} \cdots\left([a, b]^{ \pm N}\right)^{g_{m}}
$$

to be any product of conjugates for which there are as many $+N$ 's as $-N$ 's. Such an $H$ maps to the Seifert-fibered 3-manifold group

$$
\left\langle a, b, z \mid[a, b]^{N}=z^{N-1},[a, z]=[b, z]=1\right\rangle,
$$

in which $\operatorname{scl}([a, b])=(N-1) / 2 N$. The only subtle part of this last equality is the lower bound, which is certified by Bavard duality (see [Calegari 2009, Theorem 2.70]) and the existence of a rotation quasimorphism associated to a realization of the fundamental group of the Seifert manifold as a central extension of the fundamental group of a hyperbolic torus orbifold with one orbifold point of order $N$. Since scl is monotone nonincreasing under homomorphisms, the claim follows.

\section{Acknowledgements}

I would like to thank Lars Louder, Jason Manning and the anonymous referee for helpful conversations and suggestions. I would also like to thank Jason for suggesting that I call this paper "scl, SLC and SL(2, C)" and for understanding why I decided not to.

\section{References}

[Bowditch 1998] B. H. Bowditch, "Markoff triples and quasi-Fuchsian groups", Proc. London Math. Soc. (3) 77:3 (1998), 697-736. MR 99f:57014 Zbl 0928.11030

[Calegari 2008] D. Calegari, "Surface subgroups from homology", Geom. Topol. 12:4 (2008), 19952007. MR 2009d:20104 Zbl 1185.20046

[Calegari 2009] D. Calegari, scl, MSJ Memoirs 20, Mathematical Society of Japan, Tokyo, 2009. MR 2011b:57003 Zbl 1187.20035 
[Calegari and Dunfield 2006] D. Calegari and N. M. Dunfield, "An ascending HNN extension of a free group inside $\mathrm{SL}_{2} \mathbb{C} ”$, Proc. Amer. Math. Soc. 134:11 (2006), 3131-3136. MR 2007f:20046 Zbl 1159.20016

[Calegari and Walker 2011] D. Calegari and A. Walker, "Scallop", computer program, 2011, https:// github.com/aldenwalker/scallop.

[Cooper and Manning 2011] D. Cooper and J. F. Manning, "Non-faithful representations of surface groups into $S L(2, \mathbb{C})$ which kill no simple closed curve", preprint, 2011. arXiv 1104.4492

[Gabai 1983] D. Gabai, "Foliations and the topology of 3-manifolds", J. Differential Geom. 18:3 (1983), 445-503. MR 86a:57009 Zbl 0533.57013

[Gromov 1982] M. Gromov, "Volume and bounded cohomology", Inst. Hautes Études Sci. Publ. Math. 56 (1982), 5-99. MR 84h:53053 Zbl 0516.53046

[Louder 2011] L. Louder, "Simple loop conjecture for limit groups", preprint, 2011. arXiv 1106.1350

[Minsky 2000] Y. N. Minsky, "Short geodesics and end invariants", Sūrikaisekikenkyūsho Kōkyūroku 1153 (2000), 1-19. In Japanese. MR 1805224 Zbl 0968.57500

[Thurston 1986] W. P. Thurston, "A norm for the homology of 3-manifolds", pp. i-vi and 99-130 Mem. Amer. Math. Soc. 339, Amer. Math. Soc., Providence, RI, 1986. MR 88h:57014 Zbl 0585.57006

Received May 4, 2012. Revised October 3, 2012.

DANNY CALEGARI

DPMMS

UNIVERSITY OF CAMBRIDGE

CAMBRIDGE

CB3 0WA

UNITED KINGDOM

dcc43@dpmms.cam.ac.uk

http://www.its.caltech.edu/ dannyc 


\title{
PACIFIC JOURNAL OF MATHEMATICS
}

\author{
msp.org/pjm
}

Founded in 1951 by E. F. Beckenbach (1906-1982) and F. Wolf (1904-1989)

\section{EDITORS}

V. S. Varadarajan (Managing Editor)

Department of Mathematics

University of California

Los Angeles, CA 90095-1555

pacific@math.ucla.edu

Paul Balmer

Department of Mathematics

University of California

Los Angeles, CA 90095-1555

balmer@math.ucla.edu

Daryl Cooper

Department of Mathematics

University of California

Santa Barbara, CA 93106-3080 cooper@math.ucsb.edu

Jiang-Hua $\mathrm{Lu}$

Department of Mathematics

The University of Hong Kong

Pokfulam Rd., Hong Kong jhlu@maths.hku.hk
Don Blasius

Department of Mathematics University of California

Los Angeles, CA 90095-1555

blasius@math.ucla.edu

Robert Finn

Department of Mathematics Stanford University

Stanford, CA 94305-2125

finn@math.stanford.edu

Sorin Popa

Department of Mathematics

University of California

Los Angeles, CA 90095-1555

popa@math.ucla.edu

Paul Yang

Department of Mathematics

Princeton University

Princeton NJ 08544-1000

yang@math.princeton.edu

\section{PRODUCTION}

Silvio Levy, Scientific Editor, production@msp.org

\section{SUPPORTING INSTITUTIONS}

ACADEMIA SINICA, TAIPEI

CALIFORNIA INST. OF TECHNOLOGY

INST. DE MATEMÁTICA PURA E APLICADA

KEIO UNIVERSITY

MATH. SCIENCES RESEARCH INSTITUTE

NEW MEXICO STATE UNIV.

OREGON STATE UNIV.

\author{
STANFORD UNIVERSITY \\ UNIV. OF BRITISH COLUMBIA \\ UNIV. OF CALIFORNIA, BERKELEY \\ UNIV. OF CALIFORNIA, DAVIS \\ UNIV. OF CALIFORNIA, LOS ANGELES \\ UNIV. OF CALIFORNIA, RIVERSIDE \\ UNIV. OF CALIFORNIA, SAN DIEGO \\ UNIV. OF CALIF., SANTA BARBARA
}

\author{
Vyjayanthi Chari \\ Department of Mathematics \\ University of California \\ Riverside, CA 92521-0135 \\ chari@math.ucr.edu \\ Kefeng Liu \\ Department of Mathematics \\ University of California \\ Los Angeles, CA 90095-1555 \\ liu@math.ucla.edu \\ Jie Qing \\ Department of Mathematics \\ University of California \\ Santa Cruz, CA 95064 \\ qing@cats.ucsc.edu
}

These supporting institutions contribute to the cost of publication of this Journal, but they are not owners or publishers and have no responsibility for its contents or policies.

See inside back cover or msp.org/pjm for submission instructions.

The subscription price for 2013 is US \$400/year for the electronic version, and \$485/year for print and electronic.

Subscriptions, requests for back issues and changes of subscribers address should be sent to Pacific Journal of Mathematics, P.O. Box 4163, Berkeley, CA 94704-0163, U.S.A. The Pacific Journal of Mathematics is indexed by Mathematical Reviews, Zentralblatt MATH, PASCAL CNRS Index, Referativnyi Zhurnal, Current Mathematical Publications and the Science Citation Index.

The Pacific Journal of Mathematics (ISSN 0030-8730) at the University of California, c/o Department of Mathematics, 798 Evans Hall \#3840, Berkeley, CA 94720-3840, is published monthly except July and August. Periodical rate postage paid at Berkeley, CA 94704, and additional mailing offices. POSTMASTER: send address changes to Pacific Journal of Mathematics, P.O. Box 4163, Berkeley, CA 94704-0163.

PJM peer review and production are managed by EditFLOW ${ }^{\circledR}$ from Mathematical Sciences Publishers.

PUBLISHED BY

mathematical sciences publishers

nonprofit scientific publishing

http://msp.org/

(C) 2013 Mathematical Sciences Publishers 


\section{PACIFIC JOURNAL OF MATHEMATICS}

Volume $262 \quad$ No. $2 \quad$ April 2013

Certifying incompressibility of noninjective surfaces with scl

DANNY CALEGARI

Global well-posedness for the 3D rotating Navier-Stokes equations with highly oscillating initial data

QIONGLEI CHEN, CHANGXING MiAO and ZHIFEI ZHANG

Presenting Schur superalgebras

HOUSSEIN El TURKEY and JONATHAN R. KUJAWA

Classifying zeros of two-sided quaternionic polynomials and computing zeros of two-sided polynomials with complex coefficients

FENG LIANGGUI and ZHAO KAIMING

Coxeter groups, imaginary cones and dominance

XIANG FU

Semicontinuity of automorphism groups of strongly pseudoconvex domains: The low differentiability case

Robert E. Greene, Kang-Tae Kim, Steven G. Krantz and

AERYEONG SEO

Klein four-subgroups of Lie algebra automorphisms

JING-SONG HUANG and JUN YU

Fractal entropy of nonautonomous systems

Rui KuANG, WEN-ChiaO CHENG and BING LI

A GJMS construction for 2-tensors and the second variation of the total

$Q$-curvature

YoshiniKo MaTSUMOTO

Droplet condensation and isoperimetric towers

Matteo Novaga, Andrei Sobolevski and Eugene Stepanov

Brauer's height zero conjecture for metacyclic defect groups

BENJAMIN SAMBALE 